\title{
SHALLOW REEF FISH COMMUNITIES OF SOUTH BAHIA COAST, BRAZIL*
}

\author{
Laís de C. T. Chaves ${ }^{\text {*** }}$, José de Anchieta C. C. Nunes ${ }^{2}$ and Cláudio L. S. Sampaio
}

${ }^{1}$ Universidade Federal de Pernambuco

Programa de Pós-Graduação em Oceanografia

(Av. Arquitetura, $\mathrm{s} / \mathrm{n}^{\circ}$ - Cidade Universitária, 50670-901 Recife, PE, Brasil)

${ }^{2}$ Universidade Federal da Bahia

Programa de Pós-Graduação em Ecologia e Biomonitoramento

(Rua Barão de Geremoabo, s/n, 40170-280 Salvador, Bahia, Brasil)

${ }^{3}$ Universidade Federal de Alagoas

Departamento de Engenharia de Pesca

(Av. Beira Rio s/n, Centro Histórico, 57200-000 Penedo, AL, Brasil)

**Corresponding author:chaveslais@yahoo.com.br

\begin{abstract}
A B S T R A C T
This study aims to describe for the first time the fish community structure of five shallow reefs located off the cities of Santa Cruz de Cabrália and Porto Seguro (Araripe-AR, Itacipanema-IT, Alagados-AL, Naufrágio-NA and Recife de Fora Marine Park-RF Reefs), South Bahia, Northeastern Brazil. Fish density and richness were assessed through stationary point counts of $2 \mathrm{~m}$ radius for small cryptic species and $3 \mathrm{~m}$ for more conspicuous species. A total of 1802 fishes belonging to 23 families and 54 species were observed. The most abundant species were the pomacentrids Stegastes fuscus and Abudefduf saxatilis. No significant differences were found for species richness or density of conspicuous species, but density of small cryptic species was low on reefs with high bottom rugosity, where there were more holes and crevices available. Herbivores were dominant on AR, AL and $\mathrm{RF}$, invertivores on $\mathrm{AL}$ and both groups were dominant on IT. Some habitat variables such as rugosity and benthic cover were strongly correlated to species and sites. High bottom heterogeneity was found among reefs but they still presented similar species composition, richness and density. Within small-scale studies, such similarities in composition and richness are to be expected for reef fish communities, as most of the species concerned have a wide distribution range.
\end{abstract}

\section{RESUMO}

Este trabalho descreve pela primeira vez as comunidades de peixes em cinco recifes rasos de Santa Cruz de Cabrália e Porto Seguro (Araripe-AR, Itacipanema-IT, Alagados-AL, Naufrágio-NA e Recife de Fora Marine Park-RF), sul da Bahia, nordeste do Brasil. A densidade e riqueza foram obtidas utilizando censos estacionários de $2 \mathrm{~m}$ de raio para espécies pequenas e criptobênticas e $3 \mathrm{~m}$ de raio para espécies conspícuas. Um total de 1802 peixes pertencentes a 23 famílias e 54 espécies foram registrados, sendo as espécies mais abundantes dos pomacentrídeos Stegastes fuscus e Abudefduf saxatilis. Nenhuma diferença significativa foi encontrada para a riqueza entre os recifes e também para a densidade de espécies vágeis. Entretanto, a densidade de espécies criptobênticas foi mais alta em lugares com maior rugosidade. Herbívoros foram dominantes nos recifes AR, AL e RF, invertívoros em AL e a dominância dos dois grupos ocorreram em IT. Algumas variáveis ambientais como rugosidade e cobertura bentônica estão fortemente relacionadas às espécies e diferentes recifes. Estes são fisicamente heterogêneos, mas a composição e riqueza de espécies similares, o que seria esperado em comunidades recifais abrangendo estudos em pequena-escala, pois peixes recifais estão amplamente distribuídos.

Descriptors: Fish Community, Reef Ecology, Bahia Reefs

Descritores:Comunidade de peixes, Ecologia de recifes, Recifes da Bahia

\section{INTRODUCTION}

Coral reefs present the most diverse natural communities. Such ecosystems are to be compared only with tropical forests in biodiversity and are nevertheless equally threatened by human activities

(*) Paper presented at the $2^{\text {nd }}$ Brazilian Congress of Marine Biology, on 24-28 May. Búzios, RJ, Brazil. 2009.
(Prates, 2003). The most extensive reef formations of the Southwestern Atlantic (Abrolhos reef complex) are found off the northeastern coast of Brazil and provide a great diversity of reef fishes for this area (Guimarães et al., 2001).

There has been an increase in the number of Brazilian reef fish studies over the last decade. Descriptions of new species (Gasparini et al., 2001; 
Feitoza, 2002; Guimarães and Barcelar, 2002; Moura and Castro, 2002; Gasparini et al., 2003; Sampaio et al., 2004), reports of the extension of species' geographical ranges (Moura et al., 1999; Luiz Jr et al., 2008), analyses of biogeographical patterns (Floeter and Gasparini, 2000; Joyeux et al., 2001; Ferreira et al., 2004; Floeter et al., 2004, 2005, 2008) and natural history (Sazima et al., 1998; Sazima and Sazima, 2001; Feitoza et al., 2002; Nunes et al., 2007a, 2007b; Sampaio et al., 2007) are among the most prominent fields, whereas other works have been produced on reef fish community ecology, including that of coral reefs, rocky reefs and tide pools (Ferreira et al., 2001; Barreiros et al., 2004; Moura and Francini-Filho, 2005; Cunha et al., 2007; Floeter et al., 2007; Mendonça-Neto et al., 2008; Chaves and MonteiroNeto, 2009, Godinho and Lotufo, 2010).

In many tropical regions, reefs are well developed and reef fish feature as the most important source of income for traditional coastal communities. This is also true of the Bahia coast (Sampaio and Nunes, 2004), where these environments have been coming under intense pressure due to oil spills, sewage discharge, eutrophication and sedimentation (Leão and Kikuchi, 2005; Dutra et al., 2005; Floeter et al., 2006).

Bahia State presents the longest coastline in Brazil, extending for $1,188 \mathrm{~km}$. It harbors the greatest and richest coral reef area of the Southwestern Atlantic (Leão and Dominguez, 2000), but little is known of its reef fish composition and ecology. With the increasing use of the coastal zone comes the urgent need for precise information on coral reefs. Understanding reef fish community organization may provide valuable insights for the management and conservation of this ecosystem (Chittaro, 2004). Despite their historical and touristic importance, the reefs of Porto Seguro and Santa Cruz de Cabrália have remained virtually unknown until recently (Castro and Segal, 2001) and knowledge of their ichthyofauna is still sparse. This is the first study of these coastal reefs, which describes the reef fish communities of five shallow coral reefs in South Bahia. These results were accomplished through the assessment of fish species' density and richness and their association with environmental factors such as habitat complexity and benthic cover. Additional comments on fish trophic organization and distribution are made.

\section{Material ANd Methods}

Study Area

The study area comprises five coastal reefs along the coast of Porto Seguro and Santa Cruz de Cabrália municipalities, South Bahia, Brazil (Fig. 1): 1) AR-Araripe reef $\left(16^{\circ} 10^{\prime} \mathrm{S}, 38^{\circ} 54^{\prime} \mathrm{W}\right)$; 2) IT-
Itacipanema reef $\left(16^{\circ} 15^{\prime} \mathrm{S}, 38^{\circ} 57^{\prime} \mathrm{W}\right)$; 3) AL-Alagados reef $\left(16^{\circ} 16^{\prime} \mathrm{S}, 38^{\circ} 58^{\prime} \mathrm{W}\right)$; 4) NA-Naufrágio reef ( $16^{\circ} 20^{\prime}$ 'S, $\left.38^{\circ} 58^{\prime} \mathrm{W}\right)$ and, 5) RF-Recife de Fora Marine Park reef $\left(16^{\circ} 24^{\prime} \mathrm{S}, 38^{\circ} 59^{\prime} \mathrm{W}\right)$. Reefs $1-4$ never emerge at low tide. Naufrágio reef is the smallest of those studied and was thus named after the shipwreck of the 'Castor', found close to the reef. The Recife de Fora Marine Park was created in December 1997. It is located off the city of Porto Seguro, and covers an area of $17.5 \mathrm{~km}^{2}$. Depths around these reefs varied between 6 and $18 \mathrm{~m}$. In the Marine Park, water transparency is normally greater than that of the other reefs, which are closer to the coast and more affected by fluvial discharges. All the sites, except for Recife de Fora Municipal Marine Park, are characterized by high abundance of crustose and filamentous algae. Other organisms such the sea urchin Echinometra lucunter and the coral species: Siderastrea spp., Millepora spp., Mussismilia spp. and Montastrea cavernosa, are frequently observed. As for the Recife de Fora Marine Park, corals cover the greater part of the reef surface. For further details, all the reef sites studied are included in sector 3 of the Brazilian Reefs Area described by Castro and Pires (2001).

\section{Community Data}

Access was gained to the Reefs using local fishing vessels. The study was carried out in January 2007, using SCUBA diving equipment. The stationary point counts method was adapted from Bohnsack and Bannerot (1986) as in Moura (2003), where a $2 \mathrm{~m}$ radius was used for small cryptobenthic fishes (Families Labrisomidae, Cirrithidae, Grammatidae, Chaenopsidae, Gobiidae and Blenniidae) and a 3mradius was used for conspicuous species, comprising larger home range species and other site attached fish, such as territorial pomacentrids. Sampling stations were located on the reef tops. The study design included 150 diurnal samples, 30 for each reef. Records were made first for mobile species and then for cryptobenthic fish. Species density was recorded on PVC plates, each sampling taking around five minutes. An experienced observer (J.A.C.C. Nunes) was responsible for making all the counts in order to minimize any possible effect related to individual performance. Specialized literature was used for fish identification (Carvalho-Filho, 1999; Humman and DeLoach, 2003).

The species checklist is placed in order of family phylogeny following Nelson (2006). Recent changes in the classification of the Serranidae were also modified as proposed by Craig and Hastings (2007) and Smith and Craig (2007). 


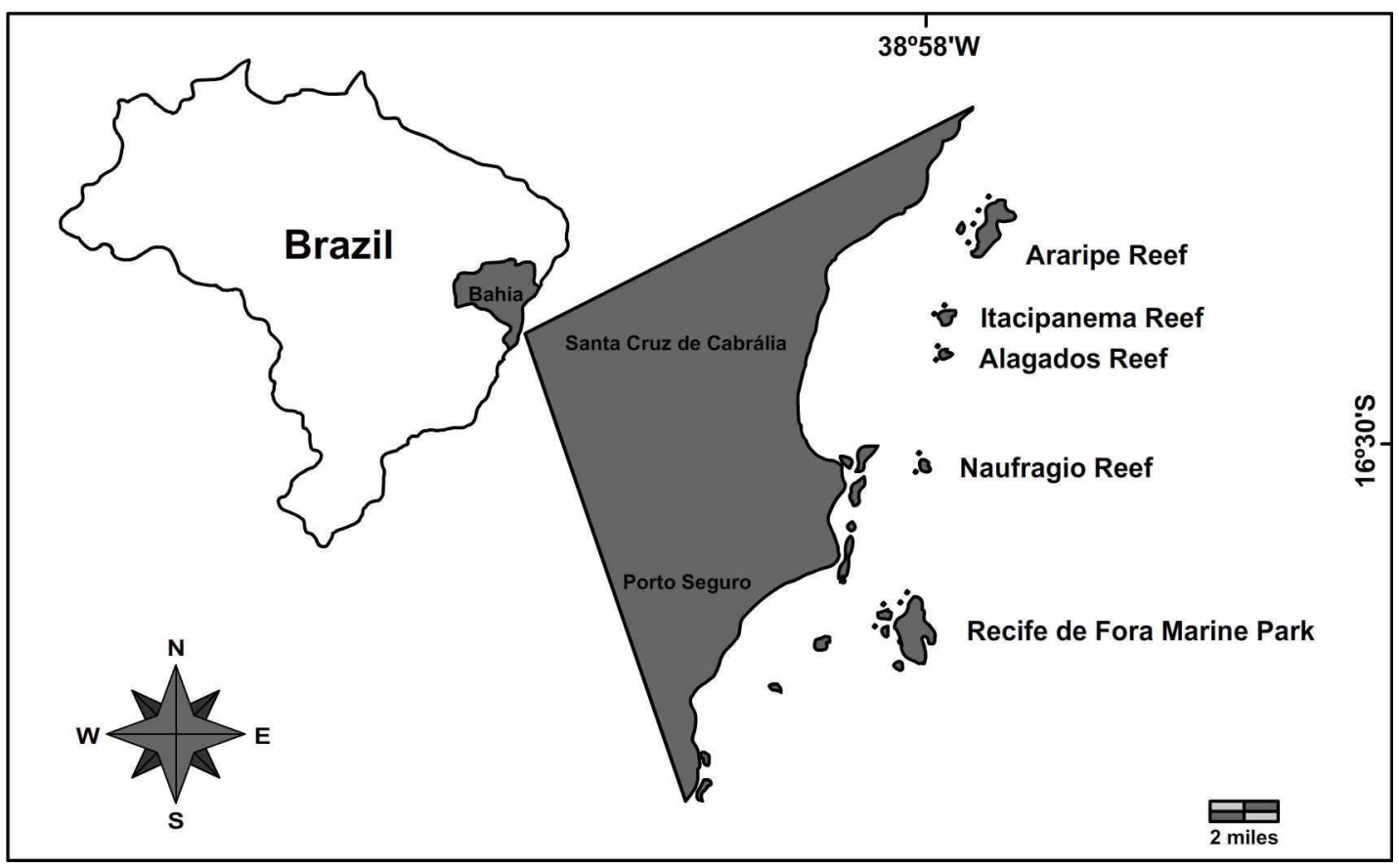

Fig. 1. Map indicating sites within the reefs studied.

Environmental Variables

Rugosity (topographical complexity) was measured using the link-chain method proposed by Luckhurst and Luckhurst (1978) as a rugosity index for each site. Depth was also measured and benthic cover obtained using replicates of a $25 \times 25 \mathrm{~cm}$ grid (100\% cover). Functional groups were used for benthic organisms (foliage algae, filamentous algae, crustose algae, corals, octocorals, sponges, echinoderms and zoantids). For each sample area two measurements of these parameters were taken at random.

Trophic Organization and Biogeographical Affinities

Trophic organization was adapted from Ferreira et al., 2004, but only 5 of their categories are used here: CAR $=$ Carnivores (which eat a variety of invertebrates and/or fishes), INV = Invertebrate feeders (feed primarily on benthic mobile and sessile invertebrates associated with the hard- or nearby softsubstrate), $\mathrm{OMN}=$ Omnivores (feed on a variety of organisms, animal and vegetal), PLK = Planktivores (feed primarily on macro- and micro-zooplankton) and HER =Herbivores (territorial and roving herbivores which include a rich mass of detritus, turf algae and/or macroalgae in their diet).

In the section Biogeographical range of the species the following codes were adopted, following
Luiz Jr. et al., 2008, where $\mathrm{Br}=$ Brazilian province (sensu Briggs 1974), CE = Central Atlantic (Islands of St. Helena and Ascension), CT = Circumtropical, SCa $=$ Southern Caribbean (Coast of Venezuela, Trinidad and Tobago and other islands of the lesser Antilles), $\mathrm{AA}=$ Amphi-Atlantic (occurring in both sides of the Atlantic Ocean), and WA $=$ Western Atlantic (occurring in both the Northern and Southern West Atlantic).

\section{Statistical Analysis}

One-Way Analysis of Variance was used to compare richness, density and physical parameters (rugosity, depth and benthic cover) between study sites. Data of conspicuous and cryptobenthic species were treated separately for the purpose of this analysis, due to the different radius sizes of the samples. The Student-Newman-Keuls (SNK) post hoc multiple comparison test was used to separate significantly different means. Data were log-transformed [log $($ density +1$)$ ] to satisfy ANOVA assumptions (Zar, 1996). Tests were performed using STATISTICA 6.0 software (Statsoft, Inc.).

Cluster analysis was used to observe patterns of similarity of fish density between study sites. Density data of all species was log-transformed and Bray-Curtis similarity was used for matrix construction. Primer 5.0 software was used for this analysis. 
Additionally, fish community and habitat relationships were analyzed using Canonical Correspondence Analysis (CCA) ordination. This analysis is a powerful technique used to understand how multiple species respond simultaneously to environmental factors, extracting significant gradients from ecological matrices (Ter Braak, 1987). Species present in less than $5 \%$ of the samples were excluded and only significant variables were used. This analysis was performed on CANOCO for Windows 4.0 (ter Braak and Verdonschot, 1995).

\section{RESULTS}

In the present study 1802 fishes belonging to 23 families and 54 species were observed (Table 1). The most abundant species are represented in Figures $1-2$.

Table 1. Species composition, abundance, geographical range and trophic category of the reef fishes recorded at Santa Cruz de Cabrália and Porto Seguro. Teleost fishes are arranged in accordance with Nelson (2006); genera and species are arranged in alphabetical order. Geographical Range: $\mathrm{AA}=\mathrm{Amphi}$-Atlantic; $\mathrm{Br}=$ Brazilian Province; $\mathrm{CT}=\mathrm{C}$ ircumtropical; EA = Eastern Atlantic; SCa =South Caribbean; WA=Western Atlantic; WA-CE=Western-Central-Eastern Atlantic. Trophic category: CAR=Carnivore; INV=Invertebrate Feeder; OMN=Omnivore; PIS=Piscivore; PLK=Planktivore; HER=Herbivore. * Cryptobenthic species; \# Species observed on dives but not included in Stationary Visual Census.

\begin{tabular}{|c|c|c|c|c|c|c|c|}
\hline Species & $\begin{array}{l}\text { Trophic } \\
\text { Category }\end{array}$ & $\begin{array}{l}\text { Biogeographic } \\
\text { Distribution }\end{array}$ & $\begin{array}{l}\text { Araripe } \\
\text { Reef }\end{array}$ & $\begin{array}{l}\text { Itacipanem } \\
\text { a Reef }\end{array}$ & $\begin{array}{l}\text { Alagados } \\
\text { Reef }\end{array}$ & $\begin{array}{l}\text { Naufrágio } \\
\text { Reef }\end{array}$ & $\begin{array}{l}\text { Recife de } \\
\text { Fora } \\
\text { Marine } \\
\text { Park }\end{array}$ \\
\hline $\begin{array}{l}\text { Muraenidae } \\
\text { Gymnothorax funebris Ranzani, } 1839 \\
\text { Gymnothorax moringa (Cuvier, } \\
\text { 1829) }\end{array}$ & $\begin{array}{l}\text { CAR } \\
\text { CAR }\end{array}$ & $\begin{array}{c}\text { WA } \\
\text { WA-CE }\end{array}$ & $0.06 \pm 0.02$ & \# & - & - & $\begin{array}{c}- \\
0.06 \pm 0.02\end{array}$ \\
\hline $\begin{array}{l}\text { Ophichthidae } \\
\text { Myrichthys ocellatus (LeSueur, 1825) }\end{array}$ & INV & WA & \# & - & $\#$ & - & $\#$ \\
\hline $\begin{array}{l}\text { Mugilidae } \\
\text { Mugil sp }\end{array}$ & OMN & $? ? ?$ & - & - & - & $\#$ & - \\
\hline $\begin{array}{l}\text { Holocentridae } \\
\text { Holocentrus adscensionis (Osbeck, } \\
\text { 1765) } \\
\text { Myripristis jacobus Cuvier, } 1829\end{array}$ & $\begin{array}{l}\text { CAR } \\
\text { PLK }\end{array}$ & $\begin{array}{l}\text { AA } \\
\text { AA }\end{array}$ & $\begin{array}{l}- \\
-\end{array}$ & $\begin{array}{c}0.13 \pm 0.02 \\
\#\end{array}$ & $\begin{array}{l}- \\
-\end{array}$ & $\begin{array}{l}- \\
-\end{array}$ & $\begin{array}{c}0.06 \pm 0.02 \\
-\end{array}$ \\
\hline $\begin{array}{l}\text { Dactylopteridae } \\
\text { Dactylopterus volitans L., } 1758\end{array}$ & INV & AA & - & \# & - & $\#$ & $\#$ \\
\hline $\begin{array}{l}\text { Scorpaenidae } \\
\text { Scorpaena plumieri Bloch, } 1789\end{array}$ & CAR & WA-CE & $\#$ & \# & $\#$ & - & - \\
\hline $\begin{array}{l}\text { Serranidae } \\
\text { Serranus flaviventris (Cuvier, 1829) }\end{array}$ & CAR & WA & \# & $\#$ & $\begin{array}{l}0.06 \pm 0.0 \\
2\end{array}$ & $0.06 \pm 0.02$ & $0.06 \pm 0.02$ \\
\hline $\begin{array}{l}\text { Epinephelidae } \\
\text { Cephalopholis fulva }(\mathrm{L} ., 1758) \\
\text { Myctoroperca bonaci (Poey, 1860) }\end{array}$ & $\begin{array}{l}\text { CAR } \\
\text { CAR }\end{array}$ & $\begin{array}{l}\text { WA } \\
\text { WA }\end{array}$ & $\begin{array}{c}0.06 \pm 0.02 \\
-\end{array}$ & - & $\begin{array}{l}\# \\
-\end{array}$ & $\begin{array}{l}\# \\
-\end{array}$ & $\begin{array}{l}\# \\
\#\end{array}$ \\
\hline $\begin{array}{l}\text { Grammatidae* } \\
\text { Gramma brasiliensis Sazima, } \\
\text { Gasparini and Moura, } 1998\end{array}$ & PLK & $\mathrm{BR}$ & - & $0.06 \pm 0.02$ & - & - & $0.06 \pm 0.02$ \\
\hline
\end{tabular}


Table 1. Continuation.

\begin{tabular}{|c|c|c|c|c|c|c|c|}
\hline Species & $\begin{array}{l}\text { Trophic } \\
\text { Category }\end{array}$ & $\begin{array}{l}\text { Biogeographic } \\
\text { Distribution }\end{array}$ & $\begin{array}{l}\text { Araripe } \\
\text { Reef }\end{array}$ & $\begin{array}{l}\text { Itacipanem } \\
\text { a Reef }\end{array}$ & $\begin{array}{l}\text { Alagados } \\
\text { Reef }\end{array}$ & $\begin{array}{c}\text { Naufrágio } \\
\text { Reef }\end{array}$ & $\begin{array}{l}\text { Recife de } \\
\text { Fora } \\
\text { Marine } \\
\text { Park }\end{array}$ \\
\hline \multicolumn{8}{|l|}{ Carangidae } \\
\hline $\begin{array}{l}\text { Carangoides bartholomaei (Cuvier, } \\
1833 \text { ) }\end{array}$ & CAR & WA & $0.06 \pm 0.01$ & - & - & - & $0.13 \pm 0.03$ \\
\hline & CAR & AA & & & $0.53 \pm 0.1$ & & \\
\hline Caranx crysos (Mitchill, 1815) & & & \# & - & 3 & - & $0.13 \pm 0.03$ \\
\hline Caranx latus Agassiz, 1831 & CAR & AA & \# & - & - & - & \# \\
\hline Caranx ruber (Bloch, 1793) & CAR & WA & - & - & \# & - & $\#$ \\
\hline $\begin{array}{l}\text { Lutjanidae } \\
\text { Lutjanus jocu (Bloch and Schneider, } \\
\text { 1801) }\end{array}$ & CAR & WA & $0.13 \pm 0.03$ & \# & \# & - & \# \\
\hline Lutjanus synagris (Linnaeus, 1758) & CAR & WA & - & - & - & $\#$ & - \\
\hline Ocyurus chrysurus (Bloch, 1791) & CAR & WA & - & - & - & - & $0.73 \pm 0.07$ \\
\hline \multicolumn{8}{|l|}{ Haemulidae } \\
\hline $\begin{array}{l}\text { Anisotremus moricandi (Ranzani, } \\
\text { 1842) }\end{array}$ & INV & $\mathrm{BR}$ & $\#$ & $0.33 \pm 0.08$ & $\#$ & - & - \\
\hline \multicolumn{8}{|l|}{ Anisotremus surinamensis (Bloch, } \\
\hline & INV & $\begin{array}{l}\text { WA } \\
\text { WA }\end{array}$ & \# & $2.00 \pm 0.30$ & $0.40 \pm 0.0$ & $0.00 \pm 0.02$ & - \\
\hline Anisotremus virginicus (L., 1758) & & & $0.13 \pm 0.02$ & $0.26 \pm 0.04$ & 4 & \# & $0.26 \pm 0.04$ \\
\hline Haemulon aurolineatum Cuvier, 1830 & INV & WA & \# & \# & $\#$ & $\#$ & $1.86 \pm 0.18$ \\
\hline Haemulon plumierii (Lacepède, 1801) & INV & WA & \# & $0.60 \pm 0.14$ & $\#$ & - & - \\
\hline \multicolumn{8}{|l|}{ Sciaenidae } \\
\hline Odontoscion dentex (Cuvier, 1830) & CAR & WA & - & $0.13 \pm 0.03$ & - & - & - \\
\hline Pareques acuminatus (Bloch and & CAR & WA & & & & & \\
\hline Schneider, 1801) & & & - & - & - & $0.06 \pm 0.02$ & $0.06 \pm 0.02$ \\
\hline \multicolumn{8}{|l|}{ Mullidae } \\
\hline $\begin{array}{l}\text { Mulloidichthys martinicus (Cuvier, } \\
1829 \text { ) }\end{array}$ & INV & AA & - & - & \# & \# & - \\
\hline $\begin{array}{l}\text { Pseudupeneus maculatus (Bloch, } \\
\text { 1793) }\end{array}$ & INV & WA & $\#$ & - & - & $0.26 \pm 0.05$ & - \\
\hline \multicolumn{8}{|l|}{ Chaetodontidae } \\
\hline Chaetodon striatus L., 1758 & INV & WA & - & \# & $\begin{array}{l}0.06 \pm 0.0 \\
2\end{array}$ & $0.06 \pm 0.02$ & $0.13 \pm 0.02$ \\
\hline \multicolumn{8}{|l|}{ Pomacanthidae } \\
\hline Holacanthus ciliaris (L., 1758) & $\begin{array}{l}\text { INV } \\
\text { OMN }\end{array}$ & $\begin{array}{l}\text { WA } \\
\text { WA }\end{array}$ & - & $0.06 \pm 0.02$ & $\stackrel{-}{-}$ & - & - \\
\hline Pomacanthus arcuatus & & & - & - & 2 & \# & - \\
\hline Pomacanthus paru (Bloch, 1787) & $\mathrm{OMN}$ & WA & $0.06 \pm 0.02$ & $0.40 \pm 0.05$ & $\#$ & $\#$ & $0.13 \pm 0.03$ \\
\hline $\begin{array}{l}\text { Cirrhitidae* } \\
\text { Amblycirrhitus pinos (Mowbray, } \\
\text { 1927) }\end{array}$ & CAR & AA & $0.06 \pm 0.02$ & - & $\begin{array}{c}0.06 \pm 0.0 \\
2\end{array}$ & - & - \\
\hline \multicolumn{8}{|l|}{ Pomacentridae } \\
\hline & OMN & $\mathrm{CT}$ & & & $3.06 \pm 0.2$ & & \\
\hline Abudefduf saxatilis (L., 1758) & & & $3.40 \pm 0.14$ & $2.60 \pm 0.14$ & 3 & $4.20 \pm 0.36$ & $0.20 \pm 0.03$ \\
\hline Microspathodon chrysurus(Cuvier, & HER & WA & & & $0.33 \pm 0.0$ & & \\
\hline 1830$)$ & HER & $\mathrm{BR}$ & $0.13 \pm 0.03$ & $0.06 \pm 0.02$ & $\begin{array}{c}5 \\
8.80 \pm 0.1\end{array}$ & $0.40 \pm 0.04$ & $0.86 \pm 0.06$ \\
\hline Stegastes fuscus (Cuvier, 1830) & & & $9.80 \pm 0.28$ & $9.00 \pm 0.20$ & 6 & $6.40 \pm 0.27$ & $9.20 \pm 0.27$ \\
\hline $\begin{array}{l}\text { Stegastes cf. variabilis (Castelnau, } \\
\text { 1855) }\end{array}$ & HER & WA & $\#$ & sor & $\#$ & $0.13 \pm 0.02$ & ser \\
\hline \multicolumn{8}{|l|}{ Labridae } \\
\hline & INV & WA & & & $0.13 \pm 0.0$ & & \\
\hline Bodianus rufus (L., 1758) & & & $0.60 \pm 0.05$ & \# & 4 & $0.06 \pm 0.02$ & - \\
\hline Halichoeres brasiliensis (Bloch, & INV & $\mathrm{BR}$ & & & $0.26 \pm 0.0$ & & \\
\hline 1791) & & & $0.33 \pm 0.03$ & $0.20 \pm 0.04$ & 4 & - & - \\
\hline Halichoeres penrosei (Starks, 1913) & INV & $\mathrm{BR}$ & - & - & - & $\#$ & - \\
\hline $\begin{array}{l}\text { Halichoeres poeyi (Steindachner, } \\
1867 \text { ) }\end{array}$ & INV & WA & $0.13 \pm 0.04$ & $1.80 \pm 0.14$ & $\begin{array}{c}0.53 \pm 0.0 \\
7\end{array}$ & $1.13 \pm 0.08$ & $0.06 \pm 0.02$ \\
\hline
\end{tabular}


Table 1. Continuation.

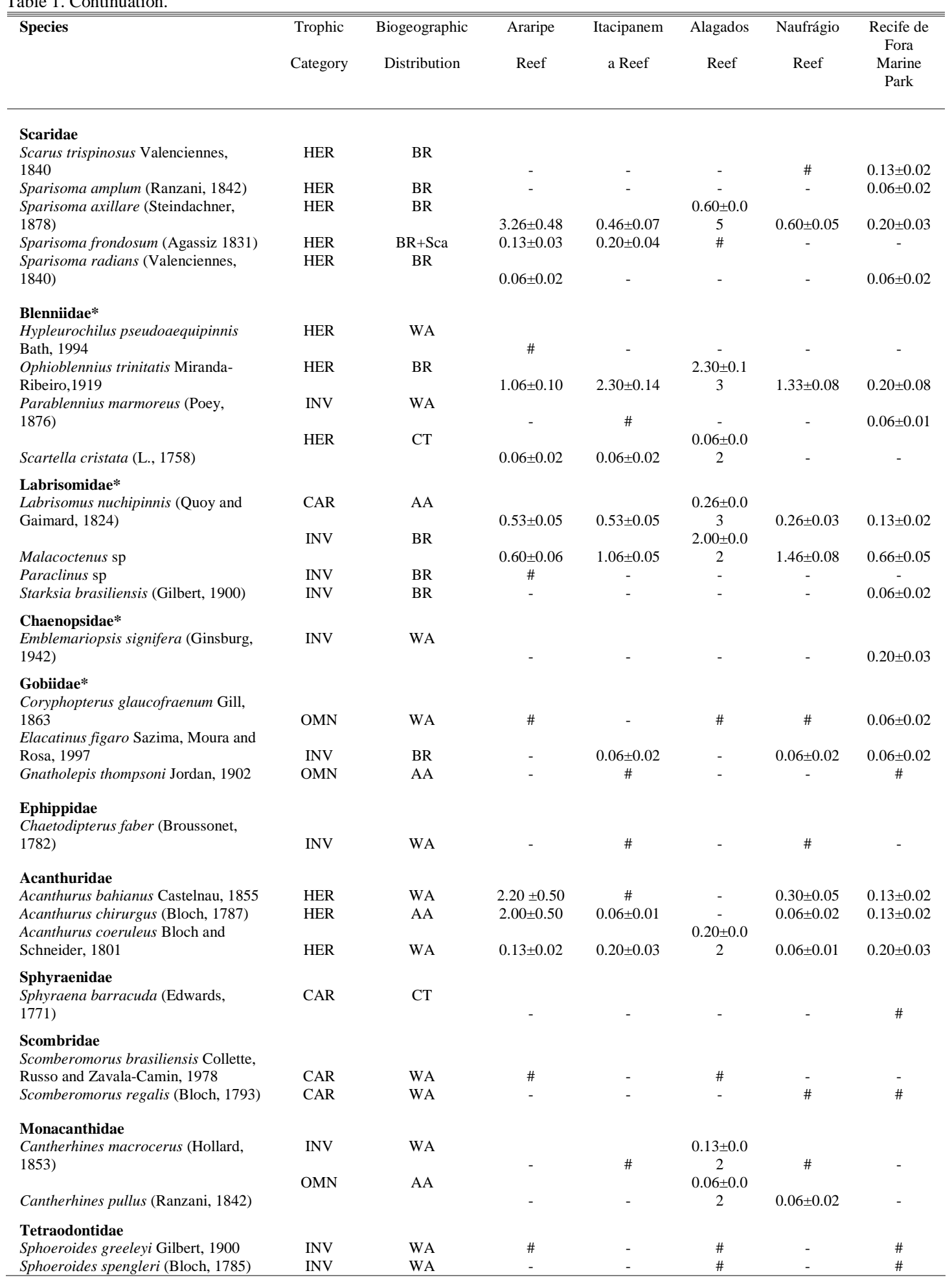




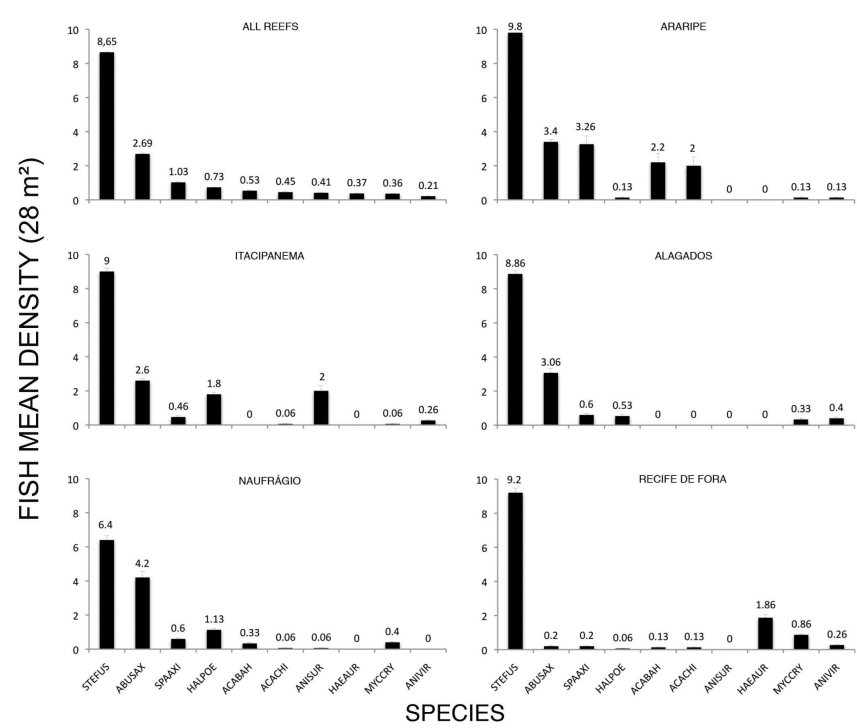

Fig. 2. Mean density per sample of conspicuous species. ( $T$ ) represents Standard Error. STEFUS = Stegastes fuscus; ABUSAX = Abudefduf saxatilis; HALPOE $=$ Halichoeres poeyi $;$ SPAAXI $=$ Sparisoma axillari; $\mathrm{ACABAH}=$ Acanthurus bahianus; ACACHI= Acanthurus chirurgus; $\mathrm{CARCRY=} \mathrm{Carangoides} \mathrm{crysos;} \mathrm{HAEPLU=}$ Haemulon plumieri $;$ HAEAUR $=$ Haemulon aurolineatum $;$ ANISUR $=$ Anisotremus surinamensis; ANIVIR= Anisotremus virginicus; $\mathrm{MICCHR}=$ Microspathodon chrysurus and OCYCHR= Ocyurus chrysurus.

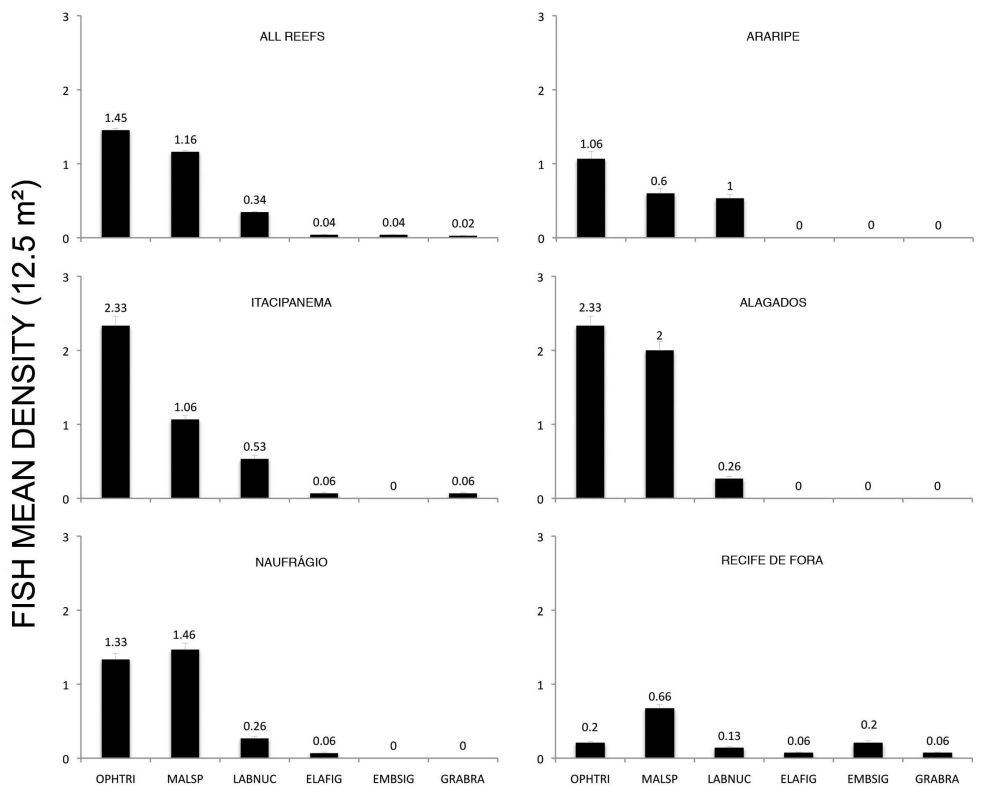

SPECIES

Fig. 3. Mean density per sample of small cryptobenthic species. $(T)$ represents Standard Error. MALSPN = Malacoctenus spn; OPHTRI= Ophioblennius trinitatis; EMBSIG = Emblemariopsis signifera $;$ LABNUC $=$ Labrisomus nuchipinnis; GRABRA= Gramma brasiliensis; ELAFIG= Elacatinus figaro $. \mathrm{AR}=$ Araripe Reef; $\mathrm{IT}=$ Itacipanema Reef, $\mathrm{AL}=$ Alagados Reef; $\mathrm{NA}=$ Naufrágio Reef and $\mathrm{RF}=\mathrm{Recife}$ de Fora Marine Park. 
No significant differences were observed for the cryptobenthic species richness between sites $(\mathrm{F}=1.24 ; \mathrm{p}=0.3)$, whereas the densities for this group differed clearly $(\mathrm{F}=3.43 ; \quad \mathrm{p}=0.02 ; \quad$ SNK: $\mathrm{Al}=\mathrm{IT}>\mathrm{AR}=\mathrm{NA}>\mathrm{RF})$. Cryptobenthic fish were more abundant on the Alagados and Itacipanema Reefs. The density and richness of conspicuous species were homogeneous for all sites $(\mathrm{F}=1.2 ; \mathrm{p}=0.15$ and $\mathrm{F}=1.91$; $\mathrm{p}=0.11$, respectively). Stegastes fuscus, Abudefduf saxatilis, Halichoeres poeyi and Sparisoma axillare were among the five most abundant species (Fig. 3).
Cluster analysis showed clearly that the Recife de Fora Marine Park formed one distinct group and the other sites formed another (Fig. 4). This result reflects the low density recorded for cryptobenthic species, due both to the reef structure of this site and the methodology applied, as will be discussed further. However, the richness was higher for this group $(n=9)$. Alagados and Naufrágio reefs were grouped together, mostly because of the lower density of S. fuscus, the most abundant on all the reefs, and the higher densities of A. saxatilis.
NAUFRÁGIO

ALAGADOS

ITACIPANEMA

ARARIPE

RECIFE DE FORA PARK

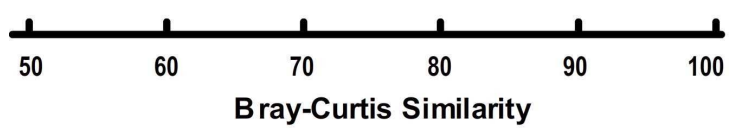

Fig. 4. Cluster Analysis indicating similarity patterns in fish abundance between sample sites. Bray-Curtis Similarity was used.

Table 2. Result of the analysis of variance carried out on the reef organisms and the physical variables considered. SNKStudent-Newman-Keuls test.

\begin{tabular}{|c|c|c|c|c|c|c|c|c|}
\hline \multirow[b]{2}{*}{$\begin{array}{l}\text { Variables } \\
(\%)\end{array}$} & \multirow[b]{2}{*}{ Araripe } & \multicolumn{3}{|c|}{ Reefs } & & \multicolumn{2}{|c|}{$\begin{array}{l}\text { ANOVA } \\
\text { Results }\end{array}$} & \multirow[t]{2}{*}{ Post-hoc SNK test } \\
\hline & & Itacipanema & Alagados & Naufrágio & $\begin{array}{l}\text { Recife de } \\
\text { Fora }\end{array}$ & $\mathrm{F}$ & $\mathrm{p}$ & \\
\hline Coral cover & $10.84 \pm 0.30$ & $5.83 \pm 0.27$ & $5.95 \pm 0.28$ & $1.26 \pm 0.28$ & $32.8 \pm 0.83$ & 15.13 & 0.00 & $\mathrm{RF}>\mathrm{AR}>\mathrm{IT}=\mathrm{AL}>\mathrm{NA}$ \\
\hline $\begin{array}{l}\text { Foliage } \\
\text { algae }\end{array}$ & $4.57 \pm 0.42$ & $17.08 \pm 0.41$ & - & $5.83 \pm 0.10$ & $3.33 \pm 0.43$ & 2.35 & $\mathrm{~ns}$ & - \\
\hline Urchins & $0.42 \pm 0.09$ & $1.67 \pm 0.07$ & $3.93 \pm 0.08$ & $2.09 \pm 0.03$ & $0.67 \pm 0.09$ & 1.08 & $\mathrm{~ns}$ & - \\
\hline Octocorals & - & $4.16 \pm 0.19$ & - & $7.91 \pm 0.14$ & - & 2.88 & 0.02 & $\mathrm{NA}>\mathrm{IT}$ \\
\hline Sponges & - & $0.42 \pm 0.18$ & - & $6.67 \pm 0.12$ & - & 0.96 & ns & - \\
\hline Zoantids & - & $24.99 \pm 0.78$ & $40.15 \pm 0.81$ & $10.41 \pm 0.19$ & $0.41 \pm 1.15$ & 8.39 & 0.00 & $\mathrm{AL}>\mathrm{IT}>\mathrm{NA}>\mathrm{RF}$ \\
\hline $\begin{array}{l}\text { Filamentous } \\
\text { algae }\end{array}$ & $25.43 \pm 0.31$ & $16.66 \pm 0.33$ & $14.95 \pm 0.38$ & $16.67 \pm 0.37$ & $47.07 \pm 0.90$ & 5.27 & 0.00 & $\mathrm{RF}>\mathrm{AR}>\mathrm{IT}=\mathrm{NA}>\mathrm{AL}$ \\
\hline $\begin{array}{l}\text { Crustose } \\
\text { algae }\end{array}$ & $58.74 \pm 1.10$ & $29.2 \pm 0.91$ & $35.01 \pm 1.02$ & $49.16 \pm 1.0$ & $15.72 \pm 1.12$ & 8.03 & 0.00 & $\mathrm{AR}>\mathrm{NA}>\mathrm{AL}>\mathrm{IT}>\mathrm{RF}$ \\
\hline Depth (m) & $2.76 \pm 0.07$ & $2.08 \pm 0.07$ & $2.45 \pm 0.07$ & $3.17 \pm 0.07$ & $3.59 \pm 0.04$ & 11.64 & 0.00 & $\mathrm{RF}>\mathrm{NA}>\mathrm{AR}=\mathrm{AL}>\mathrm{IT}$ \\
\hline $\begin{array}{l}\text { Rugosity } \\
\text { index }\end{array}$ & $1.56 \pm 0.04$ & $1.48 \pm 0.04$ & $1.51 \pm 0.04$ & $1.43 \pm 0.03$ & $2 \pm 0.02$ & 6.01 & 0.00 & $\mathrm{RF}>\mathrm{AR}=\mathrm{AL}>\mathrm{IT}=\mathrm{NA}$ \\
\hline
\end{tabular}


The fish community showed a degree of similarity for density and species richness between all the sites, but trophic composition showed some divergence (Fig.5). Herbivores are dominant in the Recife de Fora Marine Park (35.4\%) and on the Araripe $(41.1 \%)$ and Naufrágio Reefs $(40 \%)$, whereas Invertebrate feeders were dominant on the Alagados Reef $(33.5 \%)$. Both categories were balanced on the Itacipanema Reef, where they represented about $36 \%$ of the species recorded.

The Recife de Fora Marine Park and Araripe Reef presented higher numbers for carnivore species $(\mathrm{n}=8$ and 6 , respectively) whereas the others only presented 3 species each. Omnivores presented greater species richness on the Araripe $(n=6)$ and Alagados $(n=4)$ Reefs.

Some variables showed that the sites are heterogeneous (Table 2). Depth, rugosity, percentage cover of corals, octocorals, filamentous algae, zoantids and crustose algae presented significant differences, whereas echinoderms, sponges and foliage algae cover were similar among sites.

The CCA ordination diagram (Fig. 6) shows significant environmental variables represented by arrows, species by six-letter codes, and samples by symbols. The first two axes were significant $(\mathrm{p}=0.001)$ and accounted respectively for $47.4 \%$ and $30.2 \%$ of the variance between samples, species and variables.
The first axis was positively related to coral, filamentous algae and rugged habitats. This axis clearly represented RF marine park samples where both roving and territorial herbivores, represented respectively by species such as Acanthurus spp and Microspathodon chrysurus, occur in higher densities. Haemulids and Lutjanids such as Anisotremus virginicus and Lutjanus chrysurus are also more commonly found. To a lesser degree AR sites were also correlated to these parameters. The higher similarity between these reefs would be unexpected, considering the distance between them, but they cover larger surface areas and thus offer more available space to a wider range of species (e.g., large home range species). Axis 2 was positively associated with crustose algae and coral cover. Apparently, crustose algae cover was similarly distributed among reefs. Zoantids, which were negatively associated with Axis 2 were more abundant on the IT, AL and NA reefs. Abudefduf saxatilis was also more abundant on the latter reefs.

Fifty-five percent of the species occur throughout the Western Atlantic, 21\% are endemic to the Brazilian coast, $16 \%$ are Amphi-Atlantic, $4 \%$ are circumtropical, $3 \%$ are found in the Western Atlantic and the mid-Atlantic islands, Ascension and Sta. Helena, and 1\% are found in the Brazilian Province and the Caribbean.

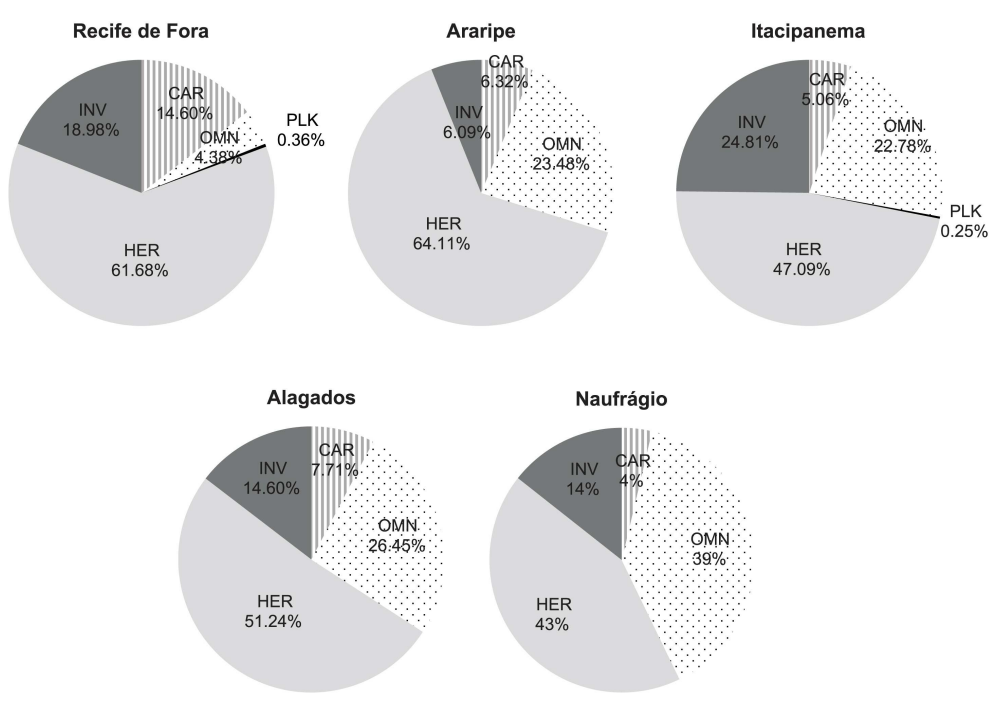

Fig. 5. Trophic composition of reef fish within reefs. INV=Invertivores, $\mathrm{CAR}=$ Carnivores, OMN=Omnivores, PLK=Planktivores, HER = Herbivores 


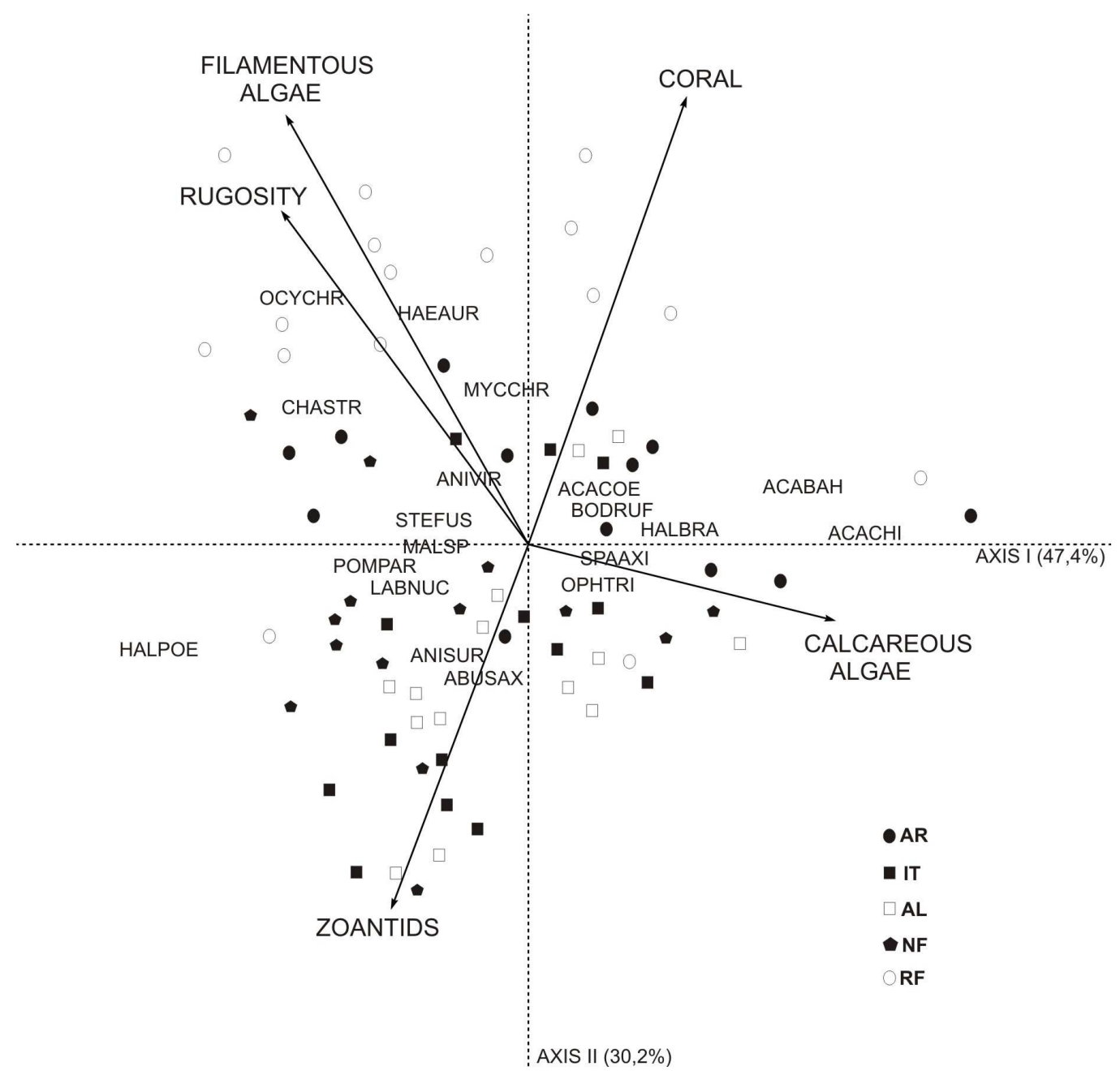

Fig. 6. Canonical Correspondence Analysis (CCA) ordination. Species are represented by six-letter codes. AR=Araripe Reef; IT=Itacipanema Reef; $A L=$ Alagados Reef; NA=Naufrágio Reef; and RF= Recife de Fora Marine Park. STEFUS = Stegastes fuscus; ABUSAX= Abudefduf saxatilis; $\mathrm{HALPOE}=$ Halichoeres poeyi; SPAAXI $=$ Sparisoma axillari; $\mathrm{ACABAH}=$ Acanthurus bahianus; $\mathrm{ACACHI}=$ Acanthurus chirurgus; CARCRY= Carangoides crysos; HAEPLU = Haemulon plumieri; HAEAUR= Haemulon aurolineatum; ANISUR = Anisotremus surinamensis; ANIVIR= Anisotremus virginicus; MICCHR= Microspathodon chrysurus; OCYCHR= Ocyurus chrysurus; BODRUF=Bodianus rufus; POMPAR=Pomacanthus paru $;$ MALSPN = Malacoctenus spn $;$ OPHTRI $=$ Ophioblennius trinitatis; HALBRA = Halichoeres brasiliensis; LABNUC $=$ Labrisomus nuchipinnis.

\section{Discussion}

Fish Community

Porto Seguro and Santa Cruz de Cabrália reefs presented lower density and richness of fishes than those reported in other studies on the reef systems of northeastern Brazil, such as Risca do Zumbi, PB (154 spp. - Feitoza, unpublished data), Tamandaré, PE (185 spp. - Ferreira et al., 2001), and especially the Abrolhos Complex, the southernmost reef formations in Bahia (Moura and Francini-Filho., 2005) and also as compared with the rocky reefs of southeastern Brazil (Ferreira et al., 1998, 2001; Floeter et al., 2006; Rangel et al., 2007). In these cases, such differences may be due to different sampling methodologies, the area covered by the respective survey, and sampling effort. Additionally, the combination of different conditions in a transitional ecological area is regarded as a major factor accounting for the high diversity in SE Brazilian reef systems (Floeter et al. 2001). 
Although shallow reefs are known to offer refuge to a great diversity and abundance of small and cryptobenthic species (Williams and Tyler, 2003), the low density of the former group in the Recife de Fora Park was unexpected. This reef presented high values for the rugosity index and coral covering, which confer habitat complexity. The record of Emblemariopsis signifera in the park, where a high coverage of braincorals (Mussismilia spp.) is found, is worth noting - such a relationship has previously been reported by Carvalho-Filho (1999).

Together with the presence of holes, habitat complexity provides more available refuge for cryptobenthic species. Ackerman and Bellwood (2000) recorded 95 individuals of 36 cryptobenthic species in a $10 \mathrm{~m}^{2}$ area of a specific location. This represented approximately $50 \%$ of the individuals and $40 \%$ of all reef fish species at this location (Depczynski and Bellwood, 2003), which demonstrates once again the limitations of methods such as the use of nondestructive visual censuses, already known to underestimate the density of cryptobenthic fishes (Smith-Vaniz et al., 2006). The methodology applied and shallow depths assessed $(\sim 2 \mathrm{~m})$ are also reasonable explanations for the lower densities of wandering species with large home ranges. Nevertheless, we assessed the more common and frequent species. Species commonly found at greater depths were excluded from the study, thus leading to an underestimation of the potential diversity expected for these reefs.

\section{Habitat Heterogeneity}

The results presented by this study show the influence of habitat complexity mainly as regards cryptobenthic species. The structure of reefs had a positive influence for richness, but as has been discussed above, underestimated their density. Several studies have examined the effects of biotic and abiotic parameters on fish community structure over extensive areas (Luckhurst and Luckhurst, 1978; Chabanet et al., 1997; Ornellas and Coutinho, 1998; Arbuto-Oropeza and Balart, 2001; Ferreira et al., 2001). Such studies regarding the Brazilian coast are increasing (Ferreira et al., 2001; Francini-Filho, 2005; Floeter et al., 2007; Chaves and Monteiro-Neto, 2009, Medeiros et al., 2010) and the great variability found suggests that the factors potentially influencing reef fish composition are highly site-dependent and variable.

Trophic Organization and Biogeographical Affinities

The reefs presented some physical heterogeneity, but their fish composition and richness were very similar. This was expected in view of the scale applied, seeing that most reef fish species are widely distributed along the coast, which reflects the high potential for dispersal among reef fishes and their ability to reach distant areas (Luiz-Jr. et al., 2008). Nevertheless, many species reported in the present study (e.g., Pomacanthus arcuatus, Anisotremus moricandi, Microspathodon chrysurus) are characteristic of the Brazilian northeastern coastal reef, but as with many of the others found, they exhibit major contributions of components of the Brazilian province.

Trophic organization in the area studied is very similar to that of the SE rocky reefs (Ferreira et al., 2001), where invertebrate feeders, herbivores and omnivores are the most abundant and speciose groups. According to Floeter et al., 2004, the density of herbivorous fishes (the dominant group relying on low-quality food resources) in the Western Atlantic decreases from tropical to temperate latitudes. The similarity observed with southeastern rocky reefs for herbivores is due to a high density of the territorial damselfish S. fuscus in the study conducted by Ferreira et al. (2001). Moreover, Moura and Francini-Filho (2005) found that carnivores were the most abundant group, followed by omnivores, herbivores and planktivore fishes in Southern Bahia. In the latter study, the trophic category for carnivores included invertebrate feeders, thus contributing to the differences observed in this study.

\section{Marine Protected Areas and Conservation Remarks}

Floeter et al. (2006) and Francini-Filho and Moura (2008b) show the effects of no-take areas on the abundance and size of reef fish as compared with those of the fishing areas on Brazilian reefs. Top predators and roving herbivores clearly presented higher abundances and sizes in areas closed to fisheries.

According to our data, the Recife de Fora Marine Park, compared to other protected reefs, presented a low abundance of top predators such as epinephelids and lutjanids, and Roving Herbivores, families of high economic value. Thus, indicating its conservation status had little or no effect as regards the protection it affords to large roving herbivores and carnivores. Although a Marine Protected Area can be a powerful tool for the replenishment of fish stocks (Francini-Filho, 2005; Francini-Filho and Moura, 2008a), it is still difficult to estimate when fish populations will attain a healthy status or whether effective management actions are being taken. Fisheries management using small-scale marine protected areas has shown positive results (e.g. McClanahan et al., 1997; Ferreira and Maida, 2001). However, only some species respond positively to protection (Micheli et al., 2005; Dulvy et al., 2004a). 
A general abundance increase of non-target species, particularly in small-sized classes, could be an indirect effect of local top predator extinction, as has been documented for several regions (Dulvy et al., 2004b; Ashworth and Ormond, 2005).

Threatened species such as Elacatinus figaro, Anisotremus moricandi and Gramma brasiliensis (MMA, 2004) reinforce the need for conservation on the reefs studied. Some efforts suggest that commercial fisheries and the ornamental fish trade has effects on the diversity and abundance of species, leading to significant changes in the local fish community structure (Costa et al., 2003; Gasparini et al., 2005; Ferreira et al., 2006). Nonetheless, past data would be needed to draw a more precise scenario. The lack of effective enforcement observed by Prates (2003) and the increasing fishing pressure inside the Park are indicative of the great need of protection in the area, that might be considered at this point in time as a mere "paper park".

This article presents a pioneer study of the composition and community structure of reef fishes in the Santa Cruz de Cabrália and Porto Seguro reefs. Baseline data are essential for biodiversity evaluations, improving our ability to predict the outcomes of alternative management strategies for reef fishes and their habitats. Given the high endemism level (10.5\%), according to Floeter et al. (2008), and a coastline extending for approximately $8,000 \mathrm{~km}$, a large-scale management plan covering the whole Marine reserves network would be an alternative for the conservation of Brazilian reef systems.

\section{ACKNOWLEDGEMENTS}

We would like to thank Camilo M. Ferreira, Ericka O. C. Coni, Rodrigo Maia-Nogueira for their intensive support during this project and Alfredo Carvalho, Luiz Rocha, Carlos E. L. Ferreira and Sergio R. Floeter for their work on the references. We would also like to thank the Centro de Ecologia e Conservação Animal (Universidade Católica do Salvador) and the Centro de Pesquisa e Conservação dos Ecossistemas Aquáticos (Biota Aquática). Financial support was provided by $\mathrm{CNPq}$ in the form of scholarships awarded to L. Chaves and J. A. Nunes.

\section{REFERENCES}

ARBUTO-OROPEZA, O.; BALART, E. F. Community structure of reef fish in several habitats of a rocky reef in the Gulf of California. Mar. Ecol., v. 22, n.4, p. 283-305, 2001.

ASHWORTH, J.S.; ORMOND, R.F.G. Effects of fishing pressure and trophic group on abundance and spillover across boundaries of a no-take zone. Biol. Conserv., v. 121, p. 333-344, 2005.
BARREIROS, J. P.; BERTONCINI, A.; Machado, L.; Hostim-Silva, M.; SANTOS, R. S. Diversity and seasonal changes in the ichthyofauna of rocky tidal pools from Praia Vermelha and São Roque, Santa Catarina. Braz. Arch. Biol. Tech., v. 47, n. 2, p. 291-299, 2004.

BONSACK, J. A.; BANNEROT, S. P. A stationary visual technique for quantitatively assessing community structure of coral reef fishes. NOAA Technical Report NMFS, v. 41, p. 1-11, 1986.

CARVALHO-FILHO, A. Peixes, Costa Brasileira. São Paulo: Melro, 3rd ed., 1999. 320 p.

CASTRO, C. B.; PIRES, D. O. Brazilian coral reefs: what we already know and what is still missing. Bull. Mar. Sci.,v. 69 , n. 2, p. 357-371, 2001

CHABANET, P.; RALAMBONDRAINY, H.; AMANIEU, M.; FAURE, G.; GALZIN, R. Relationships between coral reef substrata and fish. Coral Reefs, v. 16, p. 93$102,1997$.

CHAVES, L. C. T.; MONTEIRO-NETO, C. Comparative analysis of rocky reef fish community structure in coastal islands of south-eastern Brazil. J. Mar. Biol. Assoc. U. K., v. 89, n. 3, p. 609-619, 2009.

CHITTARO, P. M. Fish-habitat associations across multiple spatial scales. Coral Reefs, v. 23, p. 235-244, 2004.

COSTA, P. A. S.; BRAGA, A. C.; ROCHA, L. O. F. Reef fisheries in Porto Seguro, eastern Brazilian coast. Fish. Res., v. 60, p. 577-583, 2003.

CUNHA, F. E. A.; MONTEIRO-NETO, C.; NOTTINGHAM, M. C. Temporal and spatial variations in tidepool fish assemblages of the northeast coast of Brazil. Biota Neotropica, v. 7, n. 1, p. 111-118, 2007.

DULVY, N. K.; FRECKLETON, R. P.; POLUNIN, N. V. C. Coral reef cascades and the indirect effects of predator removal by exploitation. Ecol. Lett., v. 7, p. 410-416, 2004.

DUTRA, G, F.; ALLEN G, R.; WERNER T.; MCKENNA S. A. Eds. A rapid marine biodiversity assessment of the Abrolhos Bank, Bahia, Brazil. RAP Bul. Biol Assem., 38. Washington, DC: Conservation International, 2005. $160 \mathrm{p}$.

FEITOZA, B. M., ROCHA, L. A.; GASPARINI, J. L.; DIAS, T. L. First record of cleaning activity in the slippery dick, Halichoeres bivittatus. (Perciformes: Labridae), off northeastern Brazil. J. Icht. Aqu. Biol., v. 5, n. 2, p. 7376, 2002.

FEITOZA, B. M. Platygillellus brasiliensis n. sp. (Perciformes: Dactyloscopidae), the third species of the genus from the Atlantic. J. Icht. Aqu. Biol., v. 6, n. 1, p. 21-28, 2002.

FERREIRA, B.P.; MAIDA, M. Fishing and the Future of Brazil's Northeastern Reefs. InterCoast, v. 38, p. 22-23, 2001.

FERREIRA, C. E. L.; GONÇALVES, J. E. A; COUTINHO, R.; PERET, A. C. Herbivory by the Dusky Damselfish, Stegastes fuscus (Cuvier, 1830). J. Exp. Mar. Biol. Ecol., v. 229, p. 241-264, 1998.

FERREIRA, C. E. L.; GONÇALVES, J. E. A; COUTINHO, R. Fish community structure and habitat complexity in a tropical rocky shore. Environ. Biol. Fish., v. 61, p. 353369, 2001.

FERREIRA, C. E. L.; FLOETER, S. R.; GASPARINI, J. L.; JOYEUX, J. C.; FERREIRA, B. P. Trophic structure patterns of Brazilian reef fishes: a latitudinal comparison. J. Biogeogr., v. 31, p. 1093-1106, 2004. 
FLOETER, S. R.; GASPARINI, J. L. The southwestern Atlantic reef-fish fauna: composition and zoogeographic patterns. J. Fish Biol., v. 56, p. 1099-1114, 2000.

FLOETER, S. R.; FERREIRA, C. E. L.; DOMINICIAROSEMENA, A.; ZALMON, I. Latitudinal gradients in Atlantic reef fish communities: a functional approach. J. Fish Biol., v. 64, p. 1680-1699, 2004.

FLOETER, S. R.; BEHRENS, M. D; FERREIRA, C. E. L.; PADDACK, M. J.; HORN, M. H. Geographical gradients of marine herbivorous fishes: patterns and processes. Mar. Biol., v. 147, p. 1435-1447, 2005.

FLOETER, S. R.; HALPERN, B. S.; FERREIRA, C. E. L. Effects of fishing and protection on Brazilian reef fishes. Biol. Conserv., v. 128, p. 391-402, 2006.

FLOETER, S. R.; KROHLING, W.; GASPARINI, J. L.; FERREIRA, C. E. L; ZALMON, I. Reef fish community structure on coastal islands of southeastern Brazil: the influence of exposure and benthic cover. Environ. Biol. Fish., v. 78, p. 147-160, 2007.

FLOETER, S. R.; ROCHA, L. A.; ROBERTSON, D. R.; JOYEUX, J. C.; SMITH-VANIZ, W. F.; WIRTZ, P. EDWARDS, A. J.; BARREIROS, J. P.; FERREIRA, C E. L.; GASPARINI, J. L.; BRITO, A.; FALCON, J. M.; BOWEN, B. W.; BERNARDI, G. 2008. Atlantic reef fish biogeography and evolution. J. Biogeogr., v. 35, p. 22-47.

FRANCINI-FILHO, R. B.; MOURA, R. L. Dynamics of fish assemblages on coral reefs subjected to different management regimes in the Abrolhos Bank, eastern Brazil. Aquatic Conservation: Mar. Fresh. Ecos., v. 18, p. 1166-1179, 2008a.

FRANCINI-FILHO, R. B.; MOURA, R. L. Evidence for spillover of reef fishes from a no-take marine reserve: An evaluation using the before-after controlimpact (BACI) approach. Fish. Res., v. 93, p. 346-356, $2008 \mathrm{~b}$.

GASPARINI, J. L.; ROCHA, L. A.; FLOETER, S. R. Ptereleotris randalli n. sp., a new dartfish (Gobioidei: Microdesmidae) from the Brazilian Coast. J. Ich. Aq. Biol., v. 4, n.3, p. 109-114, 2001

GASPARINI, J. L.; JOYEUX, J. C.; FLOETER, S. R. Sparisoma tuiupiranga, a new species of parrotfish (Perciformes: Labroidei: Scaridae) from Brazil, with comments on the evolution of the genus. Zootaxa, v. 384 p. 1-14, 2003.

GASPARINI, J.L.; FLOETER, S.R.; FERREIRA, C.E.L.; SAZIMA, I. Marine ornamental trade in Brazil. Biodiv. Conserv., v. 14, p. 2883-2899, 2005.

GUIMARÃES, R.Z.P.; GASPARINI, J. L.; ROCHA, L. A.; FERREIRA, C. E. L.; FLOETER, S. R., RANGEL, C. A.; NUNAN, G. W. Peixes Recifais Brasileiros: Uma Riqueza Desconhecida e Ameaçada. Ciência Hoje, v. 168 , p. $16-23,2001$

GUIMARÃES, R.Z.P; BACELAR, A.C.L.H. 2002. Review of the Brazilian Species of Paraclinus (Teleostei: Labrisomidae), with Descriptions of Two New Species and Revalidation of Paraclinus rubicundus (Starks). Copeia, v. 2002, n. 2, p. 419-427. 2002.

HUMANN, P; DELOACH, N. Reef Fish Identification. New World Publication. 3rd ed. 2002. $481 \mathrm{p}$

GODINHO, W. O.; LOTUFO, T. M. Local $v$. microhabitat influences on the fish fauna of tidal pools in northeast Brazil. J. Fish biol., v. 76, p. 487-501, 2010.
JOYEUX, J. C.; FLOETER, S. R.; FERREIRA, C. E. L.; GASPARINI, J. L. Biogeography of tropical reef fish: the South Atlantic puzzle. J. Biogeogr., v. 28, p. 831841, 2001.

LEÃO, Z. M. A. N.; Dominguez, J. M. L. Tropical Coast of Brazil. Mar. Pollut. Bull., v. 41, n. 1-6, p. 112-122, 2000

LEÃO, Z. M. A. N.; KIKUCHI, R. K. P. A relict coral fauna threatened by global changes and human activities, eastern Brazil. Mar. Pollut. Bull., v. 51, p. 599-611, 2005.

LUCKHURST, B. E.; LUCKHURST, K. Analysis of influence of substrate variables on coral reef fish communities. Mar. Biol., v. 49, p.317-324, 1978.

LUIZ Jr, O. J.; CARVALHO-FILHO, A.; FERREIRA, C. E. L.; FLOETER, S. R.; GASPARINI, J. L.; SAZIMA, I. The reef fish assemblage of the Laje de Santos Marine State Park, Southwestern Atlantic: annotated checklist with comments on abundance, distribution, trophic structure, symbiotic associations, and conservation. Zootaxa (Online), v. 1807, p. 1-25, 2008.

McCLANAHAN, T.R.; GLAESEL, H.; RUBENS, J.; KIAMBO, R. The effects of traditional fisheries management on fisheries yields and the coral reef ecosystems of southern Kenya. Environ. Conserv., v. 24, p. 105-120, 1997.

MENDES, L. F. Ophioblennius trinitatis (Pisces: Blenniidae) from the oceanic archipelagos of São Pedro e São Paulo, Fernando de Noronha and Atol das Rocas. Braz. J. Ocean., v. 55, n. 1, p. 63-65, 2007.

MEDEIROS, P. R.; GREMPEL, R. G.; SOUZA, A. T. ILARRI, M. I.; R. S. Rosa. Non-random reef use by fishes at two dominant zones in a tropical, algaldominated coastal reef. Environ. Biol. Fish., v. 87, p. 237-246, 2010.

MEDEIROS, P. R.; GREMPEL, R. G.; SOUZA, A. T. ILARRI, M. I; SAMPAIO, C. L. S. Effects of recreational activities on the fish assemblage structure in a northeastern Brazilian reef. Pan. Am. J. Aq. Sci, v. 2, n. 3, p. 288-300, 2007.

MENDONÇA-NETO, J. P.; MONTEIRO-NETO, C.; MORAES, L. E. Reef fish community structure on three islands of Itaipu, Southeast Brazil. Neot. Ich., v. 6, p. 267-274, 2008.

MICHELI, F.; HALPERN, B. S.; BOTSFORD, L. W.; WARNER, R. R. Trajectories and correlates of community change in no-take marine reserves. Ecol. Appl., v. 14, p. 1709-1723, 2005.

MOURA, R. L.; GASPARINI, J. L.; SAZIMA, I. New records and range extensions of reef fishes in the western South Atlantic, with comments on reef fish distribution along the Brazilian coast. Rev. Bras. Zool., v. 16, p. 513530, 1999.

MOURA, R. L.; CASTRO, R. M. Revision of Atlantic sharpnose pufferfishes (tetraodontiformes: Tetraodontidae: Cantigaster), with description of three new species. Proc. Biol. Soc. Wash., v. 115, p. 32-50, 2002.

MOURA, R. L; FRANCINI-FILHO, R. B. Reef and shore fishes of the Abrolhos Region, Brazil. In: DUTRA, G. F.; ALLEN, G. R.; WERNER, T.; MCKENNA, S. A. (Eds). A Rapid Marine Biodiversity Assessment of the Abrolhos Bank, Brazil. Washington, Conservation International, 160 p., 2005. 
NUNES, J. A. C. C.; MAIA-NOGUEIRA, R.; SAMPAIO, C L. S. "Friendly behavior'" between two species of Myrichthys in Brazilian waters. Coral Reefs, v. 26, p. $199,2007$.

NUNES, J. A. C. C.; CHAVES, L. C. T.; MAIANOGUEIRA, R.; SAMPAIO, C. L. S. Association between small fishes and the Carribean Reef Squid Sepiotheutis sepioidea (Blainville, 1923) (Teuthida:Lolinginidae). J. Mar. Biol. Assoc. U. K., v. 87, p. 1-2, 2007.

NUNES, J. A. C. C; SAMPAIO, C. L. S. Notes on the ecology of the poorly know Brownstriped grunt, Anisotremus moricandi (Ranzani, 1842), in the coastal reefs of the Northeastern region of Brazil. Pan. Am. J. Aq. Sci., v. 2, n. 1, p. 2, 2007.

ORNELLAS, A. B.; COUTINHO, R. Spatial and temporal patterns of distribution and abundance of a tropical fish assemblage in a seasonal Sargassum bed, Cabo Frio Island, Brasil. J. Fish Biol., v. 53(A), p.198-208, 1998.

PRATES, A. P. Atlas dos recifes de corais nas unidades de conservação do Brasil. MMA/SBF, v. 1, 2003. 180 p.

RANGEL C. A.; CHAVES, L. C. T.; MONTEIRO-NETO, C. Baseline assessment of the reef fish assemblage from Cagarras Archipelago, Rio de Janeiro, southeastern Brazil. Braz. J. Oceanogr., v. 55, n. 1, p. 7-17, 2007.

ROCHA, L.A. Patterns of distribution and processes of speciation in Brazilian reef fishes. J. Biogeogr., v. 30, p. 1161-1171, 2003.

SAMPAIO, C. L. S.; NUNES, J. A. C. C.; MENDES, L. Acyrtus pauciradiatus., a new species of clingfishes (Teleostei: Gobiesocidae) from Fernando de Noronha Archipelago, Pernambuco state, Northeast Brazil. Neot. Ich., v. 2, n. 4, p. 205-208, 2004.
SAZIMA, I.; MOURA, R. L.; GASPARINI, J. L. The wrasse Halichoeres cyanocephalus (Labridae) as a specialized cleaner fish. Bull. Mar. Sci., v. 63, n. 3, p. 605-610, 1998.

SAZIMA, C.; SAZIMA, I. Plankton-feeding aggregation and occasional cleaning by adult butterflyfish, Chaetodon striatus (Chaetodontidae), in southwestern Atlantic. Cybium, v. 25, n. 2, p. 145-151, 2001.

SMITH-VANIZ, W. F.; JELKS, H. L.; ROCHA, L. A. Relevance of cryptic fishes in biodiversity assessments: a case study at Buck island reef national monument, St. Croix. Bull. Mar. Sci., v. 79, n. 1, p. 17-48, 2006.

TER BRAAK C.F. Ordination. In JONGMAN H., TER BRAAK C.J. and VAN TONGEREN O.F. (Eds) Data analysis in community and landscape ecology. Wageningen, The Netherlands: Backhuys Publishers, 1987. p. 91-173.

TER BRAAK, C. J. F.; VERDONSCHOT, P. F. M. Canonical correspondence analysis and related multivariate methods in aquatic ecology. Aquat. Sci., v. 57, p. 255-289, 1995.

WILLIAMS, J. T.; TYLER, J. C. 2003. Revision of western Atlantic clingfish of the Tomicodon (Gobiesocidae), with descriptions of five new species. Smithsonian Contrib. Zool., v. 621, 26p.

ZAR, J. H. Biostatistical analysis. $4^{\text {th }}$ ed. New Jersey: Prentice-Hall, 1996. 663p.

(Manuscript received 04 June 2009; revised 14 July 2010; accepted 06 August 2010) 